\title{
Características agronómicas de la soya en función de las densidades de siembra y profundidad de deposición de abono ${ }^{1}$
}

\author{
Jorge Wilson Cortez 2 , Carlos Eduardo Angeli Furlani', Rouverson Pereira da Silva ${ }^{3}$, \\ Rodrigo Alberto Alandia Román ${ }^{4}$
}

\section{RESUMEN}

El objetivo de este trabajo fue evaluar las características agronómicas de la soya sembrada en Sistema de Siembra Directa, en función de las densidades de siembra (15, 16 y 20 plantas por metro) y la profundidad de deposición de abono (11, 14 y $17 \mathrm{~cm}$ ). El experimento fue realizado en el Departamento de Ingeniería Rural, UNESP/Jaboticabal, utilizando, en el delineamiento, parcelas subdivididas en bloques al acaso, con cuatro repeticiones. Características agronómicas de la soya analizadas: número de días de aparición, índice de aparición, porcentaje de daños, altura de plantas, altura de inserción de la primera vaina, vainas por planta, granos por vaina, porcentaje de plantas sobrevivientes y productividad. Los resultados indicaron que el número de días de aparición, índice de aparición y el porcentaje de daños, no fueron influenciados por las densidades y la profundidad de deposición de abono. Los daños por insectos y aves fueron del orden de $30 \%$ de plantas atacadas. La altura de plantas, altura de inserción de la primera vaina, vainas por planta y granos por vaina no variaron en relación a la densidad y profundidad de deposición de abono. La productividad de la soya $\left(5.146 \mathrm{~kg} \mathrm{ha}^{-1}\right)$ fue semejante en las densidades y profundidades de deposición de abono.

Palabras-clave: Productividad, vainas por planta, población, profundidad de abono, Glycine max L. Merr.

\section{RESUMO}

\section{Características agronômicas da soja em função das densidades de semeadura e profundidade de deposição do adubo}

O objetivo deste trabalho foi avaliar as características agronómicas da soja semeada em Sistema de Plantio Direto, em função das densidades de semeadura (15, 16 e 20 plantas por metro) e da profundidade de colocação do adubo (11, 14 e $17 \mathrm{~cm}$ ). O experimento foi realizado no Departamento de Engenharia Rural, UNESP/Jaboticabal, utilizando, no delineamento, parcelas subdivididas em blocos ao acaso, com quatro repetições. Características agronômicas da soja analisadas: número de días para emergência, índice de emergência, porcentagem de danos, altura de plantas, altura de inserção da primeira vagem, número de vagens por planta, grãos por vagem, porcentagem de plantas sobreviventes e produtividade. Os resultados indicaram que o número de días para emergencia, índice de emergencia e porcentagem de danos não foram influenciados pelas densidades de plantio e profundidade de aplicação dos adubos. Os danos por insetos e aves atingiram valores de $30 \%$ de plantas atacadas. A altura das plantas, altura de inserção da primeira vagem, número de vagens por planta e de grãos por vagem não variaram em função da densidade de plantio e profundidade de aplicação do adubo. A produtividade da soja $\left(5.146 \mathrm{~kg} \mathrm{ha}^{-1}\right)$ foi similar nas densidades de plantio e profundidade de deposição do adubo.

Palavras-chave: Produtividade, vagens por planta, população, profundidade de adubação, Glycine max L. Merr.

\footnotetext{
Recibido para publicacion en julio del 2009 y aprobado en enero del 2011

'Extraido de la Disertación de Máster (Producción Vegetal) del primer autor

${ }^{2}$ Ing. Agrónomo, Doctor. UNIVASF - Universidade Federal do Vale do São Francisco, Rodovia Br 407, 12, Lote 543, 56300-000 - BA, Brasil, jorge.cortez@univasf.edu.br

${ }^{3}$ Ing. Agrónomo, Doctor, UNESP - Universidade do Estado de São Paulo, Rodovia Paulo D. Castellane, s/n, 14884-900 - Jaboticabal, SP, Brasil. furlani@fcav.unesp.br rouverson@fcav.unesp.br

${ }^{4}$ Ing. Agrónomo, Estudiante de Máster, UNESP, Rodovia Paulo D. Castellane, s/n, 14884-900 - Jaboticabal, SP, Brasil. pulpo_alandia@hotmail.com
} 


\section{INTRODUCCIÓN}

El término Sistema de Siembra Directa (SSD) surgió, según Dallmeyer (2001), en el ámbito de la Federación de Asociaciones de Plantío Directo de la Paja, y con el uso popular consagró la nomenclatura. El autor cita que la operación ejecutada es la siembra directa, pero que involucra a todo el sistema cuanto a diversos equipamientos y máquinas que lo apoyan, pudiendo ser con el uso de trituradores, rodillo de cuchillas y pulverizadores.

El uso del SSD se caracteriza por la menor intensidad de movilización del suelo y por la reducción de la frecuencia del tráfico de máquinas sobre el terreno, y por mantener sobre la superficie del suelo gran cantidad de cobertura vegetal, proveniente de un cultivo implantado para ese fin, siendo imprescindible el uso de cobertura, por el uso de rotación. La utilización en el SSD de soya, ayudó a expandir las áreas con actividad agrícola, principalmente debido a la capacidad de esa en corregir errores en la regulación de la sembradora.

Gassen (2004) cita que en suelos compactados, surco limpio y exceso de fertilizantes en el surco, bien debajo de la semilla, se dificultará el contacto suelo/semilla para absorción de agua y el desarrollo de raíces. Para el autor, lo ideal es hacer la siembra con el surco preparado más profundo y la semilla más superficial con mejor acabamiento por las ruedas compactadoras.

Así, para que haya el crecimiento, desarrollo y rendimiento de la soya es necesaria la interacción entre el potencial genético de un determinado cultivo con el ambiente. En las condiciones del campo, la naturaleza proporciona la mayor parte de las influencias ambientales sobre el desarrollo, no obstante, los productores, por medio de las prácticas de tratamiento ya comprobadas, pueden manipular el ambiente de producción (Suzuki et al., 2005). Para el autor, la productividad de la soya en la década de los 70 era del orden de $1.800 \mathrm{~kg} \mathrm{ha}^{-1}$, en la década de los 80 aumentó hasta $2.400 \mathrm{~kg} \mathrm{ha}^{-1}$, en la década de 90, debido a las inversiones en investigación, alcanzó $3.000 \mathrm{~kg} \mathrm{ha}^{-1}$ y en el año 2005, el nivel de productividad está arriba de $3.600 \mathrm{~kg} \mathrm{ha}^{-1} \mathrm{y}$ se espera llegar hasta $4.200 \mathrm{~kg} \mathrm{ha}^{-1}$.

Herzog et al. (2004) constataron que aun aumentando la profundidad del cincel surcador de abono (6 para 12 $\mathrm{cm})$ no encontraron diferencia en la productividad de la soya que fue de 2.944 y $3.047 \mathrm{~kg} \mathrm{ha}^{-1}$, respectivamente. Lo mismo ocurrió para la masa seca de la parte aérea de la soya que produjo 2.313 y $2.481 \mathrm{~kg} \mathrm{ha}^{-1}$ para las profundidades de 6 y $12 \mathrm{~cm}$, respectivamente.

Trabajos con densidades de siembra no han mostrado efecto en el rendimiento de granos, inclusive variando de 8 hasta 63 plantas por $\mathrm{m}^{2}$ (Rubin, 1997; Pires et al., 1998). La inexistencia de respuesta diferenciada para rendimiento de granos a la variación de la densidad de siembra de la soya está íntimamente relacionada con la plasticidad fenotípica que este cultivo presenta (Rambo et al., 2003). De acuerdo con Tourino et al. (2002), el porcentaje de sobrevivencia de las plantas aumenta a medida que ocurre reducción de la densidad de siembra, debido a la competición intraespecífica de las plantas y a que por el aumento de la densidad de siembra, las alturas fueron menores, probablemente por ser una característica genética.

Ludwig et al. (2007), al estudiar tres densidades de siembra de soya, $250.000,400.000$ y 550.000 plantas por hectárea verificaron aumento en la producción con el incremento de la densidad, por otro lado, el número de vainas por planta disminuyó con el aumento de las densidades. Los autores también verificaron que el número de granos por vaina y la masa de granos no variaron en función de las densidades.

El objetivo general de la preparación de este trabajo es la obtención de resultados cuanto número de días de aparición, índice de aparición, porcentaje de daños, altura de plantas, altura de inserción de la primera vaina, vainas por planta, granos por vaina, porcentaje de plantas sobrevivientes y productividad, en diferentes densidades de siembra en el cultivo de la soya y profundidades de deposición de abono en la siembra directa.

\section{MATERIALES Y MÉTODOS}

El experimento fue realizado en el área experimental del Departamento de Ingeniería Rural perteneciente a la UNESP, Jaboticabal, Estado de San Pablo, localizada en las coordenadas geodésicas: latitud $21^{\circ} 14^{\prime} \mathrm{S}$ y longitud $48^{\circ} 16^{\prime} \mathrm{W}$, con altitud promedio de 559 m.s.n.m., declividad promedio de $4 \%$, ocupando un área de aproximadamente 1,0 ha, en el periodo de noviembre/2005 a marzo/2006.

La siembra fue realizada en Latosuelo Rojo Eutroférrico típico (Dark Red Latossol - Haplustox) en Tabla 1 A, moderado, textura arcillosa y relieve suave ondulado (EMBRAPA, 2006). El suelo se encontraba con humedad promedio de $23 \%$ en la camada de 0 a $20 \mathrm{~cm}, 73 \%$ de cobertura vegetal de maíz, obtenida por medio de la metodología de Laflen et al. (1981), y resistencia a penetración de 1,7 y 3,3 MPa en las camadas de 0 a 10 y 10 a $20 \mathrm{~cm}$, respectivamente.

El clima, de acuerdo con la clasificación de Köppen (1948), es Cwa, o sea, subtropical húmedo, con sequía en el período del invierno. En la Tabla 2, se pueden verificar los datos metereológicos durante la realización del experimento.

Fueron utilizados para la siembra un tractor $73,6 \mathrm{~kW}$ (100 cv en la rotación de $2.300 \mathrm{rpm}$ ) de potencia en el motor, con masa de $5.400 \mathrm{~kg}$ y $40 \%$ de lastre en el eje 
delantero (neumáticos 23.1-26R1 de 4,9 $\mathrm{m}$ de perímetro y presión de 18 psi (124 KPa)), y 60\% de lastre en el eje trasero (neumáticos 23.1 - 26 R1 de 4,9 m de perímetro y presión de inflado de $22 \mathrm{psi}(152 \mathrm{KPa})$ ).

Sembradora-abonadora de precisión con siete líneas de siembra (separadas en $50 \mathrm{~cm}$ ), dotada de discos de corte de paja de $45,7 \mathrm{~cm}$, cincel surcador de abono con las siguientes características: $2,7 \mathrm{~cm}$ de espesura de puntera, $1,0 \mathrm{~cm}$ de espesura del cincel, distancia del disco de corte al cincel de $12 \mathrm{~cm}$, relación entre la altura y el largo de la puntera $(\mathrm{H} / \mathrm{L})$ de 1,06 y ángulo de ataque de $20^{\circ}$; disco doble desencontrado de 40,6 cm para deposición de semilla. La máquina posee distribuidor de semillas a turbina, siendo utilizado disco de semillas de 64 orificios. La distribución del abono fue realizada por mecanismo helicoidal. El depósito de abono posee capacidad para 1.310 $\mathrm{kg}$ y el de semilla para $200 \mathrm{~kg}$, teniendo la sembradora $3.070 \mathrm{~kg}$ de masa, trabajando con $665 \mathrm{~kg}$ de abono en la operación de siembra.

Antes de la siembra fue realizada una aplicación de Glifosato con dosis de $4 \mathrm{~L} \mathrm{ha}^{-1}$ y posteriormente otra aplicación con 1,5 $\mathrm{L} \mathrm{ha}^{-1}$ de 2,4 $\mathrm{D}$, ambos con tasa de aplicación de $120 \mathrm{~L} \mathrm{ha}^{-1}$. Fueron utilizadas semillas de soya de la variedad Monsoy 5942 (Superprecoz) que fueron tratadas con $300 \mathrm{~mL}$ de Thiram $700 \mathrm{SC}$ (carboxinthiram) para $100 \mathrm{~kg}$ de semilla e inoculante líquido con dosis de $500 \mathrm{~mL}$ para $50 \mathrm{~kg}$ de semilla. Para el control de malezas después de la siembra, se utilizó $600 \mathrm{~mL} \mathrm{ha}^{-1}$ de Flex (nitrobenzamida); $600 \mathrm{~mL} \mathrm{ha}^{-1}$ de Fusilade (fluzifop-p-butil); Raptor (imazamox) $70 \mathrm{~g} \mathrm{ha}^{-1}$ y $200 \mathrm{~mL} \mathrm{ha}^{-1}$ de aceite mineral, siendo dos pulverizaciones con tasa de aplicación de $250 \mathrm{~L} \mathrm{ha}^{-1}$. Para el control de chinches fue utilizado Endosulfan $350 \mathrm{CE}$, con dosis de 1,5 $\mathrm{L} \mathrm{ha}^{-1}$, con tasa de aplicación de $300 \mathrm{~L} \mathrm{ha}^{-1}$. Para Roya Asiática fue hecha una aplicación con fungicida Opera (epoxiconazole) siendo $500 \mathrm{~mL} \mathrm{ha}{ }^{-1}$, con tasa de aplicación de $300 \mathrm{~L} \mathrm{ha}^{-1}$ y una segunda aplicación con Priori Xtra (azoxistrobina e ciproconazole) con dosis de $300 \mathrm{~mL} \mathrm{ha}^{-1}$. Para las enfermedades de final de ciclo, se hizo una aplicación de Rival 200 EC (tebuconazole) con dosis de $300 \mathrm{~mL} \mathrm{ha}^{-1}$ con tasa de aplicación de $300 \mathrm{~L} \mathrm{ha}^{-1}$.

El diseño experimental utilizado fue el de parcelas subdivididas en bloques al azar con cuatro repeticiones. Los tratamientos fueron: las densidades de siembra de 15, 16 y 20 plantas por metro, siendo esas densidades obtenidas por medio de variaciones en los engranajes de cambio rápido de la sembradora; la profundidad de deposición del abono regulada en 11, 14 y $17 \mathrm{~cm}$ con auxilio de la rueda de control de profundidad del cincel surcador de abono. Las parcelas recibieron, como tratamiento princi-

Tabla 1. Fertilidad del Latosuelo Rojo Eutroférrico típico y análisis granulométrico del suelo en la camada de 0 a $20 \mathrm{~cm}$

\begin{tabular}{|c|c|c|c|c|c|c|c|c|c|c|}
\hline \multirow[b]{2}{*}{ Tratamiento } & \multicolumn{10}{|c|}{ Fertilidad del suelo } \\
\hline & pH & M.O. & $P$ resina & $\mathbf{K}$ & $\mathbf{C a}$ & Mg & $\mathbf{H}+\mathbf{A l}$ & SB & $\mathbf{T}$ & $\mathbf{V}$ \\
\hline & $\mathrm{CaCl}_{2}$ & $\%$ & $\mathrm{mg} \mathrm{dm}^{-3}$ & \multicolumn{4}{|c|}{$\mathrm{mmol}_{\mathrm{c}} \mathrm{dm}^{-3}$} & \multicolumn{3}{|c|}{$\%$} \\
\hline \multirow[t]{4}{*}{ Promedio } & 5,4 & 2,98 & 54 & 4,2 & 40 & 21 & 29 & 65 & 99 & 67 \\
\hline & \multicolumn{10}{|c|}{ Granulométrica del suelo } \\
\hline & \multirow[t]{2}{*}{ Arcilla } & \multirow{2}{*}{\multicolumn{2}{|c|}{ Limo }} & & \multicolumn{3}{|c|}{ Arena } & \multirow{3}{*}{\multicolumn{3}{|c|}{ Clase Textural }} \\
\hline & & & & & fina & & gruesa & & & \\
\hline \multirow{2}{*}{ Muestra } & \multicolumn{7}{|c|}{$\%$} & & & \\
\hline & 55 & & 25 & & 10 & & 10 & \multicolumn{3}{|c|}{ Arcillosa } \\
\hline
\end{tabular}

MO: materia orgánica: SB; suma de bases; V: saturación de bases del suelo; T: capacidad de cambio

Tabla 2. Datos meteorológicos del local del experimento

\begin{tabular}{|c|c|c|c|c|c|c|c|}
\hline Mes & $\begin{array}{c}\text { Presión } \\
(\mathbf{h P a})\end{array}$ & $\begin{array}{c}\text { Tmax } \\
\left({ }^{\circ} \mathrm{C}\right)\end{array}$ & Tmin & $\begin{array}{c}\text { Tmed } \\
\left({ }^{\circ} \mathrm{C}\right)\end{array}$ & HR & ND & $\begin{array}{c}\text { Insolación } \\
\text { (h) }\end{array}$ \\
\hline \multicolumn{8}{|c|}{2005} \\
\hline Octubre & 942,5 & 32,7 & 20,0 & 25,2 & 66,6 & 10 & 222,3 \\
\hline Noviembre & 941,0 & 31,4 & 18,8 & 24,3 & 67,6 & 7 & 220,2 \\
\hline Diciembre & 941,0 & 29,5 & 19,2 & 23,5 & 77,7 & 19 & 204,3 \\
\hline \multicolumn{8}{|c|}{2006} \\
\hline Enero & 942,3 & 31,3 & 20,3 & 25 & 74,7 & 18 & 2336 \\
\hline Febrero & 941,1 & 30,7 & 20,3 & 24,2 & 82,9 & 15 & 181,1 \\
\hline Marzo & 941,9 & 31 & 20,4 & 24,5 & 81,4 & 16 & 221 \\
\hline
\end{tabular}

Presión: presión atmosférica; Tmax: temperatura máxima; Tmin: temperatura mínima; Tmed: temperatura promedio; HR: Humedad relativa del aire; ND: número de días con lluvia. 
pal, las densidades de siembra y las subparcelas (tratamiento secundario) recibieron las profundidades de deposición del abono. Las parcelas medían $65 \mathrm{~m}$ de largo por 3,15 m de ancho, con $15 \mathrm{~m}$ de intervalo para maniobras y estabilización del conjunto.

Fueron recolectados datos de aparición de las plántulas hasta la estabilización del número de plantas que surgieron. El número promedio de días para aparición fue determinado por medio de la ecuación utilizada por Edmond \& Drapala (1958).

$$
M=\frac{[(N 1 \cdot G 1)+(N 2 \cdot G 2)+\ldots+(N n \cdot G n)]}{(G 1+G 2+\cdots+G n)}
$$

en la cual:

M: número promedio de días para la aparición de las plántulas de soya;

$\mathrm{N}_{1}$ número de días entre la siembra y el primer conteo de plántulas;

$\mathrm{G}_{1}$ : número de plántulas que surgieron en el primer conteo;

$\mathrm{N}_{2}$ : número de días entre la siembra y el segundo conteo de plántulas;

$\mathrm{G}_{2}$ : número de plántulas que aparecieron entre el primer y segundo conteo;

$\mathrm{N}_{\mathrm{n}}$ : número de días entre la siembra y el último conteo de plántulas, y

$\mathrm{G}_{\mathrm{n}}$ : número de plántulas que aparecieron entre el penúltimo y el último conteo.

El índice de germinación es un factor de desempeño de las sembradoras de siembra directa y se ha optado por eso, con base en la relación entre la cantidad de semillas distribuidas y la cantidad de plántulas que aparecieron en el área explotada, o sea, la eficiencia de la máquina en relación al establecimiento del cultivo (Portella et al., 1997).

Los daños causados a las plantas que surgieron fueron medidos en un metro de la parcela al final de su establecimiento, por el conteo del número de plantas damnificadas o cortadas por aves, hormigas y diversos insectos del suelo.

En la determinación de la población inicial y final, fue utilizado un pedazo de madera de un metro en el cual fue colocado un cable, también de madera, conectado a él, en el centro, formando una " $T$ " invertida. En el campo fue colocada la parte inicial de este equipo, junto a la planta de soya y se efectuó el conteo del número de plantas en este espacio, los conteos fueron realizados en la hilera central de cada parcela. El porcentaje de sobrevivencia fue calculado por la diferencia entre la población inicial y final.

La determinación de altura final de plantas fue efectuada por el conteo de 10 plantas de la parcela y los valores fueron expresados en promedio por parcela. Las determinaciones de altura de inserción de la primera vaina (AIPV) fueron efectuadas por el conteo de éstas en 10 plantas por parcela. La altura de la primera vaina fue medida por medio de una punta de madera con una cinta métrica fija, graduada de $1 \mathrm{~cm}$, medido del nivel del suelo hasta la inserción de la primera vaina. La determinación del número de vainas fue efectuada por el conteo de las mismas en 10 plantas.

El número de granos por vaina fue calculado dividiendo el número total de granos de la planta por la cantidad de vainas encontradas en la misma. La masa de 100 granos fue obtenida por el pesaje de una muestra recolectada aleatoriamente de los granos cosechados de las diez plantas de cada parcela y corregidos para $13 \%$ de humedad. La masa seca fue obtenida por medio del pesaje de 10 plantas de cada parcela experimental, posteriormente transformadas en $\mathrm{kg} \mathrm{ha}^{-1}$.

Fueron recolectadas 10 plantas consecutivas de la hilera central de cada parcela, descontando las orillas, correspondiendo a $50 \mathrm{~cm}$ en cada extremidad. Después de la recolección de las plantas, éstas fueron trilladas y pesadas separadamente por parcela y sus valores corregidos para $13 \%$ de humedad.

Los datos obtenidos fueron tabulados y sometidos al análisis de variación. Cuando el valor de la prueba de F fue significativo a $5 \%$ de probabilidad, fue realizado la prueba de Tukey para la comparación de promedios. Los datos obtenidos fueron analizados con ayuda del software ESTAT (UNESP, 1991).

\section{RESULTADOS Y DISCUSIÓN}

El número de días para aparición indica la cantidad de días que las plántulas demoraron para salir del suelo. El índice de aparición (semillas viables propuestas en la regulación/plantas que aparecieron) es uno de los parámetros para evaluar el conjunto dosificadordepositador de semillas de una sembradora-abonadora, y el porcentaje de daños causados por animales que inhiben el crecimiento inicial de las plántulas, siendo esos parámetros presentados en la Tabla 3.

No se encontró diferencia en los parámetros presentados para los tratamientos, visto que las condiciones de siembra y las climáticas fueron adecuadas para un óptimo establecimiento y aparición de plántulas.

El índice de aparición es un parámetro de eficiencia de la máquina en relación a la cantidad de plantas propuesta en la regulación, y aun no habiendo diferencia en el análisis, cuando se aumentó la densidad, se exige más del sistema depositador, ocurriendo disminución en el IE de $16 \%$ en relación a la menor densidad. Según Portella et al. (1997), las sembradoras dotadas de disco liso para corte 
de paja, cincel surcador para abono y disco doble desencontrado para semillas, presentaron los mejores resultados para el índice de aparición.

Los daños por determinados tipos de aves e insectos existen en gran cantidad; con el objetivo de presentar cuanto ese daño puede ser grave, los resultados promedios quedaron entre $30 \%$ para todos los tratamientos.

Población inicial, final y porcentaje de plantas sobrevivientes son presentados en la Tabla 4. La población inicial, por la regulación de la sembradora, tendría que distribuir aproximadamente 15,19 y 24 semillas por metro respectivamente para las D1, D2 y D3, lo que no ocurrió, debido a posibles fallas de la sembradora para esas densidades. Si consideramos el índice de aparición, y adicionamos $19 \%$ para la densidad de 20 semillas por metro, se obtiene el resultado esperado de plantas que fue propuesto; considerando el índice de sobrevivencia, para la densidad de 15 y 16 semillas por metro, se observó una reducción de $1,8 \%$ y $15,7 \%$ en las densidades, respectivamente. Para la profundidad de deposición de abono, no se observa diferencia entre los tratamientos y para los parámetros en la Tabla 4.

La población final de plantas se diferenció apenas para la mayor densidad, indicando que, con el aumento, se consigue obtener densidades que son indicadas por los productores de semillas.

En la Tabla 4 son presentados los datos de altura de plantas y altura de inserción de la primera vaina en la maduración. No se observó diferencia significativa en-

Tabla 3. Síntesis del análisis de variación cuanto al número de días para aparición (NDA), índice de aparición (IE) y porcentaje de daños (D)

\begin{tabular}{lccc}
\hline Factores & NDA & IE $(\%)$ & D $(\%)$ \\
\hline Densidad (plantas por metro) & & & \\
\hline 15 & $6,0 \mathrm{a}$ & $97,2 \mathrm{a}$ & $28,3 \mathrm{a}$ \\
16 & $6,3 \mathrm{a}$ & $83,8 \mathrm{a}$ & $33,1 \mathrm{a}$ \\
20 & $6,2 \mathrm{a}$ & $81,6 \mathrm{a}$ & $29,5 \mathrm{a}$ \\
\hline Profundidad (cm) & & & \\
\hline 11 & $6,3 \mathrm{a}$ & $88,1 \mathrm{a}$ & $34,5 \mathrm{a}$ \\
14 & $6,0 \mathrm{a}$ & $87,2 \mathrm{a}$ & $28,9 \mathrm{a}$ \\
17 & $6,2 \mathrm{a}$ & $87,2 \mathrm{a}$ & $27,5 \mathrm{a}$ \\
\hline Prueba F & & & \\
\hline Densidad (D) & $0,4^{\mathrm{NS}}$ & $2,6^{\mathrm{NS}}$ & $0,2^{\mathrm{NS}}$ \\
Profundidad (P) & $0,4^{\mathrm{NS}}$ & $0,1^{\mathrm{NS}}$ & $0,3^{\mathrm{NS}}$ \\
D x P & $0,4^{\mathrm{NS}}$ & $1,6^{\mathrm{NS}}$ & $0,6^{\mathrm{NS}}$ \\
\hline C.V.D & 9,8 & 20,9 & 63,8 \\
\hline C.V.P & 10,9 & 10,9 & 74,2 \\
\hline Pom & & & \\
\end{tabular}

Promedios seguidos de la misma letra en la columna no difieren por la prueba de Tukey. ${ }^{\text {NS }}$. No significativo $(\mathrm{p} \geq 0,05)$; $^{*}$ : significativo $(\mathrm{p}<$ $0,05) ;{ }^{* *}$; significativo $(\mathrm{p}<0,01)$ : C.V.: coeficiente de variación $(\%)$. tre los tratamientos propuestos. Contrariando la afirmación de que el desarrollo de las plantas puede ser modificado por la densidad de plantas, Ballaré et al. (1995) constataron eso en parte, por mecanismos que usan informaciones sobre la luz del ambiente, por medio de fotosensores específicos. Esos resultados pueden ser confrontados con los de CORTEZ (2004) que trabajando con soya, variedad Vencedora, obtuvo promedio de 17 $\mathrm{cm}$ para la altura de inserción de la primera vaina, valores mayores cuando comparados a este experimento, probablemente por trabajar con una variedad de ciclo largo, al paso que de este experimento la variedad es de ciclo superprecoz.

Tourino et al. (2002) constataron que, con el aumento de la densidad de plantas, las alturas fueron menores, lo que no quedó observado en este experimento, probablemente en razón de que esa altura es una característica genética. No obstante, Costa \& Tavares (1995) afirmaron que la densidad de siembra menor trae plantas más bajas y vainas cercanas al suelo.

En la Tabla 5 son presentados los datos para número de vainas por planta, granos por vaina y productividad. Se puede observar que los parámetros no fueron afectados por los tratamientos, lo que no era de esperarse, visto que, según Balleré et al. (1995), cuando se utilizan varias densidades de siembra el desarrollo de las mismas es alterado. Por otro lado, Ludwig et al. (2007) encontraron diferencia en la productividad con el aumento de la densidad y reducción del número de vainas por planta.

Según Rambo et al. (2003), el número de vainas es el componente del rendimiento que más sufre modificaciones por la utilización de prácticas diferenciadas de manipulación con las densidades de siembra, lo que no fue verificado en este experimento, aun ocurriendo variaciones de $25 \%$.

En este experimento no se verificó diferencia cuanto al número de granos por vaina, lo que concuerda con Board et al. (1990) y Rambo et al. (2003) quienes observaron el número de granos por vaina no siendo afectado por las densidades de siembra, debido a que este componente es determinado en el final del ciclo reproductivo de la soya. El número de granos por vaina tiene control genético sustancial y por eso tiene pequeña variación (IOWA, 1994).

Según Tourino et al. (2002) la variación de la densidad afectó significativamente el número de vainas por planta, pero no hubo efecto significativo sobre el número de granos por vaina. El número de vainas por planta varió inversamente a la variación de la densidad de las plantas, o sea, la reducción de la densidad provocó aumento en el número de vainas por planta, lo que no fue verificado. 
Se observó que, en la producción, los factores fueron independientes y no influenciaron la producción de granos del cultivo de la soya. La producción encontrada en este experimento de $5.148 \mathrm{~kg} \mathrm{ha}^{-1}$, viene a confirmar lo que Suzuki et al. (2005) dicen sobre las nuevas variedades de soya que llegarían a producir más de $4.200 \mathrm{~kg} \mathrm{ha}^{-1}$.

La utilización de densidades entre 15 y 20 plantas por metro no modificaron la producción, concordando con Tourino et al. (2002) que verificaron en densidades entre 10 y 15 plantas por metro, comparadas con las mayores, no modificaron la producción y ayudaron a disminuir los gastos con la compra de semilla por ocasión de siembra, y que las menores poblaciones compensan la producción por la mayor cantidad de ramos laterales.

Rambo et al. (2003), trabajando con diferentes densidades de siembra, no encontraron diferencia entre las mismas, obteniendo promedio de $4.500 \mathrm{~kg} \mathrm{ha}^{-1}$ en la productividad de la soya.

Copetti (2003) afirma que la soya soporta variaciones de hasta $15 \%$ en la densidad de siembra sin alterar la productividad, sin embargo, variaciones de $25,5 \%$ ocurridas en este experimento, tampoco alteraron la productividad.

Tabla 4. Síntesis del análisis para población inicial y final (EI y EF = Mil semillas ha ${ }^{-1}$ ), plantas sobrevivientes (PS), altura de plantas en la maduración (AP) y de inserción de la primera vaina (AIPV)

\begin{tabular}{|c|c|c|c|c|c|}
\hline Factores & $\mathbf{E I}$ & $\mathbf{E F}$ & $\operatorname{PS}(\%)$ & $\mathbf{A P}(\mathbf{c m})$ & AIPV $(\mathbf{c m})$ \\
\hline \multicolumn{6}{|c|}{ Densidad (plantas por metro) } \\
\hline 15 & $323,9 \mathrm{~b}$ & $246,3 \mathrm{~b}$ & $77,3 \mathrm{a}$ & $57,5 \mathrm{a}$ & $8,5 \mathrm{a}$ \\
\hline 16 & $353,5 a b$ & $290,7 \mathrm{~b}$ & $81,2 \mathrm{a}$ & $57,3 \mathrm{a}$ & $8,9 \mathrm{a}$ \\
\hline 20 & $435,1 \mathrm{a}$ & $362,9 \mathrm{a}$ & $84,2 \mathrm{a}$ & $53,1 \mathrm{a}$ & $9,3 \mathrm{a}$ \\
\hline \multicolumn{6}{|c|}{ Profundidad (cm) } \\
\hline 11 & $370,2 \mathrm{a}$ & $244,4 \mathrm{a}$ & $80,8 \mathrm{a}$ & $55,5 \mathrm{a}$ & $8,7 \mathrm{a}$ \\
\hline 14 & 368,4 a & $311,1 \mathrm{a}$ & 83,7 a & $55,8 \mathrm{a}$ & $9,3 \mathrm{a}$ \\
\hline 17 & $373,9 \mathrm{a}$ & $296,3 \mathrm{a}$ & $79,3 \mathrm{a}$ & $56,5 \mathrm{a}$ & $8,7 \mathrm{a}$ \\
\hline \multicolumn{6}{|l|}{ Prueba F } \\
\hline Densidad (D) & $6,8^{*}$ & $19,3^{*}$ & $0,3^{\mathrm{NS}}$ & $2,1^{\mathrm{NS}}$ & $2,7^{\mathrm{NS}}$ \\
\hline Profundidad (P) & $0,1^{\mathrm{NS}}$ & $0,8^{\mathrm{NS}}$ & $0,7^{\mathrm{NS}}$ & $0,5^{\mathrm{NS}}$ & $1,7^{\mathrm{NS}}$ \\
\hline $\mathrm{D} \times \mathrm{P}$ & $1,5^{\mathrm{NS}}$ & $1,8^{\mathrm{NS}}$ & 1,7 Ns & $1,4^{\mathrm{NS}}$ & $0,9^{\mathrm{NS}}$ \\
\hline C.V.D & 20,6 & 15,6 & 28,6 & 10,7 & 9,8 \\
\hline C.V.P & 11,1 & 12,5 & 13,9 & 4,36 & 10,68 \\
\hline
\end{tabular}

Promedios seguidos de la misma letra en la columna no difieren por la prueba de Tukey. ${ }^{\text {Ns: }}$ No significativo ( $\mathrm{p} \geq 0,05$ ); ${ }^{*}:$ significativo (p $\leq 0,05) ;{ }^{* *}$ : significativo $(\mathrm{p} \leq 0,01) ;$ C.V.: coeficiente de variación $(\%)$.

Tabla 5. Análisis de variación para vainas por planta, granos por vaina, y productividad

\begin{tabular}{|c|c|c|c|}
\hline Factores & Vainas por planta & Granos por vaina & Productividad $\left(\mathrm{kg} \mathrm{ha}^{-1}\right)$ \\
\hline \multicolumn{4}{|c|}{ Densidad (plantas por metro) } \\
\hline 15 & $53 \mathrm{a}$ & $2,8 \mathrm{a}$ & $5.208 \mathrm{a}$ \\
\hline 16 & $57 \mathrm{a}$ & $2,8 \mathrm{a}$ & $5.502 \mathrm{a}$ \\
\hline 20 & $49 \mathrm{a}$ & $2,6 \mathrm{a}$ & $4.728 \mathrm{a}$ \\
\hline \multicolumn{4}{|c|}{ Profundidad (cm) } \\
\hline 11 & $57 \mathrm{a}$ & $2,7 \mathrm{a}$ & $5.634 \mathrm{a}$ \\
\hline 14 & $50 \mathrm{a}$ & $2,7 \mathrm{a}$ & $4.800 \mathrm{a}$ \\
\hline 17 & $51 \mathrm{a}$ & $2,8 \mathrm{a}$ & $5.004 \mathrm{a}$ \\
\hline \multicolumn{4}{|l|}{ Prueba F } \\
\hline Densidad (D) & $1,0^{\mathrm{NS}}$ & $2,6^{\mathrm{NS}}$ & $0,7^{\mathrm{NS}}$ \\
\hline Profundidad (P) & $2,1^{\mathrm{NS}}$ & $1,2^{\mathrm{NS}}$ & $3,3^{\mathrm{NS}}$ \\
\hline $\mathrm{D} \times \mathrm{P}$ & $2,1^{\mathrm{NS}}$ & $1,4^{\mathrm{NS}}$ & $2,3^{\mathrm{NS}}$ \\
\hline C.V.D & 28,3 & 7,0 & 31,0 \\
\hline C.V.P & 17,1 & 7,1 & 16,0 \\
\hline
\end{tabular}

Promedios seguidos de la misma letra en la columna no difieren por la prueba de Tukey. Ns: No significativo ( $\mathrm{p} \geq 0,05$ ); ${ }^{*}$ : significativo (p $\leq 0,05) ;{ }^{* *}$ : significativo $(\mathrm{p} \leq 0,01) ;$ C.V.: coeficiente de variación $(\%)$. 


\section{CONCLUSIONES}

El número de días para aparición, índice de aparición y el porcentaje de daños, no fueron influenciados por las densidades y la profundidad de deposición de abono.

Las poblaciones inicial y final son afectadas por la densidad de siembra.

La altura de plantas, altura de inserción de la primera vaina, vaina por planta y granos por vaina no variaron en relación a la densidad y la profundidad de deposición del abono.

La productividad de la soya fue semejante en las densidades y profundidades de deposición del abono.

\section{REFERENCIAS}

Ballaré CL, Scopel, AL \& Sánchez RA (1995). Plant photomorphogenesis in canopies, crop growh, and yield. Hort Science, 30:1172-1181.

Board JE, Harville BG \& Saxton AM (1990) Narrow-row seedyield enhancement indeterminate soybean. Agronomy Journal, 82:64-68.

Copetti E (2003). Plantadoras: Distribuição de sementes. Cultivar Máquinas, 18:14-17.

Costa NP \& Tavares LCV (1995). Fatores responsáveis pelos elevados percentuais de perdas de grãos durante a colheita mecânica da soja. Informativo ABRATES, 5:17-25.

Cortez JW (2004). Avaliação de semeadora-adubadora em coberturas, manejos e velocidades de semeadura na cultura da soja. Monografia de Graduação em Agronomia, Faculdades Associadas de Uberaba, FAZU, Uberaba, 62p.

Dallmeyer AU (2001) Equipamentos: semeadura. Cultivar Máquinas, 2:6-9.

Edmond JB \& Drapala WL (1958). The effects of temperature, sand and soil acetone on germination of okra seed. Proceedings of American Society Horticulture Science, 71:428-34.

Empresa Brasileira de Pesquisa Agropecuária - EMBRAPA (2006). Centro Nacional de Pesquisas de Solos. Sistema Brasileiro de Classificação de Solos. Brasília, 306p.

Gassen DN (2004). Manejo: problemas com semeadura sob plantio direto. Plantio Direto, 84:51-52.

Herzog RL, Levien R \& Trein CR (2004). Produtividade de soja em semeadura direta influenciada por profundidade do sulcador de adubo e doses de resíduo em sistema irrigado e não irrigado. Engenharia Agrícola, 24:771-780.

Iowa State University (1994). How a soybean plant develops. Iowa State University, Cooperative Extension Service, Ames, Iowa, USA. 20p.

Köppen WP (1948). Climatologia, con un estudio de los climas de la tierra. México: Fondo de Cultura Económica. 478p.

Laflen JM, Amemiya M \& Hintz EA (1981). Measuring crop residue cover. Journal of Soil and Water Conservation, 36:341343.

Ludwig MP, Dutra LMC, Zabot L, Jauer A, Uhry D, Farias JR, Losekann ME, Stefanelo C, Lucca Filho OA (2007). Efeito da densidade de semeadura e genótipos no rendimento de grãos e seus componentes na soja semeada após a época indicada. $\mathrm{Re}$ vista da FZVA, 14:13-22.
Pires JL, Costa JA \& Thomas AL (1998). Rendimento de grãos de soja influenciado pelo arranjo de plantas e níveis de adubação. Pesquisa Agropecuária Gaúcha, 4:183-188.

Portella JA, Sattler A \& Faganello A (1997). Desempenho de elementos rompedores de solo sobre o índice de emergência de soja e de milho em plantio direto no sul do Brasil. Engenharia na Agricultura, 5:209-217.

Rambo L, Costa JA, Pires JLF, Parcianello G \& Ferreira FG (2003). Rendimento de grãos da soja em função do arranjo de plantas. Ciência Rural, 33:405-411.

Rubin, SAL (1997). Comportamento do cultivar "FEPAGRO-RS 10 " em seis densidades de semeadura no planalto médio riograndense. In: Reunião de Pesquisa de Soja da Região Sul, 25, Passo Fundo. Anais... EMBRAPA: Passo Fundo, Brasil. CDROM.

Suzuki S, Yuyama MM \& Camacho SA (2005). Boletim de Pesquisa de Soja. Rondonópolis: Fundação Mato Grosso: Campo Grande. 230 p.

Tourino MCC, Rezende PM \& Salvador N (2002). Espaçamento, densidade e uniformidade de semeadura na produtividade e características agronômicas da soja. Pesquisa Agropecuária Brasileira, 37:1071-1077.

Universidade Estadual Paulista - UNESP (1991). Sistema para análises estatísticas: ESTAT. V. 2.0. Jaboticabal, UNESP.

Rev. Ceres, Viçosa, v. 58, n.1, p. 62-68, jan/fev, 2011 